\title{
CRESCIMENTO DA CULTURA DO ARROZ SUBMETIDO À OMISSÃO DE NUTRIENTES EM LATOSSOLOS DO ESTADO DO PARÁ
}

\author{
Juliana Souza da Silva ${ }^{1}$; José Darlon Nascimento Alves²; Morieli Ladislau de Oliveira ${ }^{3}$; \\ Danielly Cristina da Silva Marques ${ }^{4}$; Marcus Alberto Ribeiro Macêdo ${ }^{5}$; \\ Eduardo Cézar Medeiros Saldanha ${ }^{6}$.

\footnotetext{
${ }^{1}$ Universidade Federal Rural da Amazônia. Capitão Poço, Pará, Brasil. ac_ju@ hotmail.com ${ }^{2}$ Universidade Federal Rural da Amazônia. Capitão Poço, Pará, Brasil. jose.darllon@ @otmail.com ${ }^{3}$ Universidade Federal Rural da Amazônia. Capitão Poço, Pará, Brasil. morieliladislau@ @otmail.com ${ }^{4}$ Universidade Federal Rural da Amazônia. Capitão Poço, Pará, Brasil. marquesdanielly@ hotmail.com ${ }^{5}$ Universidade Federal Rural da Amazônia. Capitão Poço, Pará, Brasil. albert.marcus @ hotmail.com
} \\ ${ }^{6}$ Universidade Federal Rural da Amazônia. Capitão Poço, Pará, Brasil. saldanha@ufra.edu.br
}

\begin{abstract}
RESUMO: O objetivo do trabalho foi avaliar o efeito da omissão de macro e micronutrientes no crescimento de plantas de arroz em Latossolos de textura média e argilosa. $\mathrm{O}$ experimento foi instalado na casa de vegetação da Universidade Federal Rural da Amazônia (UFRA), no município de Belém/PA. Os tratamentos avaliados foram: T1) Controle; T2) Completo $($ Calagem + macronutrientes + B, Zn, Cu, Si, Ni); T3) NPK + B, Zn, Cu, Mn + Si (sem calagem); T4) Omissão de N; T5) Omissão de P; T6) Omissão de K; T7) Omissão de Ca; T8) Omissão de Mg; T9) Omissão de B, Zn, Cu, Si, Ni; T10) Omissão de Si; e T11) Omissão de Ni. Foram utilizados dois substratos (LATOSSOLO AMARELO de textura média e argilosa). A deficiência do fósforo ocasionou diretamente menor crescimento da planta, no LATOSSOLO AMARELO de textura média. Enquanto, a omissão de potássio no LATOSSOLO AMARELO de textura argilosa proporcionou menor crescimento, o que sugere que a limitação desses nutrientes ocasionou restrições no desenvolvimento das plantas.
\end{abstract}

PALAVRAS-CHAVE: deficiência, nutrição de plantas, Oryza sativa.

\section{GROWTH OF RICE CULTURE SUBMITTED TO OMISSION OF NUTRIENTS IN THE STATE OF PARA OXISOLS}

\begin{abstract}
The present study aims to evaluate the effect of the omission of macro and micronutrients in the growth of rice plants in sandy loam Oxisols and clayey. The experiment was conducted in a greenhouse at the Federal Rural University of Amazonia (UFRA) in the city of Belém / PA. The treatments were: T1) Control; T2) Complete (Liming + macronutrients + B, Zn, Cu, Si, Ni); T3) NPK + B, Zn, Cu, Mn + Si (without lime); T4) N omission; T5) P omission; T6) K omission; T7) Ca omission; T8) Mg omission; T9) Omission of $\mathrm{B}, \mathrm{Zn}, \mathrm{Cu}, \mathrm{Si}, \mathrm{Ni}$; T10) Si omission; and T11) Ni omission. In two substrates (OXISOL texture medium and clay). A deficiency of phosphorus caused directly lower plant growth in OXISOL medium texture. While the omission of potassium in the OXISOL clayey of provided less growth, which suggested that the limitation of these nutrients caused restrictions on plant development.
\end{abstract}

KEYWORDS: deficiency, Oryza sativa, plant nutrition. 
A deficiência nutricional provoca a diminuição do crescimento das plantas em decorrência de alterações na eficiência nutricional, constituindo aspecto pouco abordado na literatura que trata da omissão de nutrientes em plantas (GONDIM et al., 2010), uma vez que a baixa eficiência das adubações nitrogenadas, além de limitar a produtividade e aumentar o custo de produção, pode ocasionar problemas de poluição ambiental. Assume, portanto, grande importância à adequação do sistema produtivo, visando à elevação da eficiência de utilização de nitrogênio pelo arroz, mediante o aprimoramento das práticas de manejo (SCIVITTARO, 2006).

A utilização de doses, épocas de aplicação (parcelamento ou não) e fontes de $\mathrm{N}$ podem aumentar significativamente a eficiência do uso dos fertilizantes nitrogenados e, consequentemente, a produtividade de culturas anuais, como o arroz (FAGERIA et al., 2003).

De maneira geral, a dose de $\mathrm{N}$ a ser utilizada na cultura baseia-se na produtividade almejada e no histórico da área. Aliado a este fato, a crescente utilização de cultivares de alto potencial produtivo tem implicado no uso frequente de insumos, dentre os quais $\mathrm{O} \quad \mathrm{N}$ (HERNANDES et al., 2010).

A queda de produtividade do arroz em várias regiões do mundo está relacionada com vários fatores, sendo uma delas a baixa disponibilidade de nutrientes ou de elementos benéficos (MAUAD et al., 2003). Dessa forma, o objetivo do trabalho foi avaliar o efeito da omissão de macro e micronutrientes no crescimento de plantas de arroz em Latossolos de textura média e argilosa.

$\mathrm{O}$ experimento foi instalado na casa de vegetação da Universidade Federal Rural da Amazônia (UFRA), no município de Belém - PA (012 27' 21" S 48 30' 14" O).

Foram utilizados substratos de diferentes composições, sendo coletados em locais distintos. O LATOSSOLO AMARELO de textura média foi coletado no município de Capitão Poço - PA e o LATOSSOLO AMARELO de textura argilosa foi retirado do município de Paragominas - PA, na profundidade de 0$20 \mathrm{~cm}$, apresentando as seguintes características químicas e físicas do solo (Tabela 1).

Utilizou-se o delineamento experimental inteiramente casualizado com 11 tratamentos, dois substratos de diferentes texturas (média e argilosa), com três repetições. Totalizando 66 unidades experimentais, sendo que cada unidade estava constituída por cinco plantas por vaso.

Os tratamentos avaliados foram: T1) Controle; T2) Completo (Calagem + macronutrientes $+\mathrm{B}, \mathrm{Zn}, \mathrm{Cu}, \mathrm{Si}, \mathrm{Ni}$ ); T3) $\mathrm{NPK}+\mathrm{B}, \mathrm{Zn}, \mathrm{Cu}, \mathrm{Mn}+\mathrm{Si}$ (sem calagem); 
T4) Omissão de N; T5) Omissão de P; T6)

Omissão de K; T7) Omissão de Ca; T8)

Omissão de Mg; T9) Omissão de B, Zn,
$\mathrm{Cu}, \mathrm{Si}, \mathrm{Ni}$; T10) Omissão de Si; e T11) omissão de Ni.

Tabela 1. Características das análises químicas e físicas do solo da área experimental, provenientes da camada de 0-0,20 $\mathrm{m}$ de profundidade, coletadas em LATOSSOLO AMARELO de textura média e LATOSSOLO AMARELO de textura argilosa.

\begin{tabular}{|c|c|c|c|c|c|c|c|c|c|}
\hline Local & $\mathrm{pH}$ & $\mathrm{P}$ & $\mathrm{K}$ & $\mathrm{Ca}$ & $\mathrm{Mg}$ & $\mathrm{Al}$ & $\mathrm{H}+\mathrm{Al}$ & SB & CTC \\
\hline & $\mathrm{H}_{2} \mathrm{O}$ & - $\mathrm{mg} \mathrm{dm}$ & $-3---$ & & & $--c^{-1}$ & $\mathrm{dm}^{-3}-$ & & \\
\hline Capitão Poço & 4,2 & 1,37 & 14 & 1,0 & 0,2 & 0,45 & 3,15 & 1,12 & 4,27 \\
\hline Paragominas & 3,9 & 0,98 & 32 & 0,6 & 0,4 & 1,00 & 4,62 & 1,08 & 5,70 \\
\hline & V & $\mathrm{m}$ & Areia & Silte & Argila & \multicolumn{4}{|c|}{ Textura } \\
\hline & ------ & $\%$-------- & ------י n & $--\mathrm{g} \mathrm{kg}$ & $-\cdots$ & \multirow{3}{*}{\multicolumn{4}{|c|}{ Franco arenosa }} \\
\hline Capitão Poço & 26,2 & 28,6 & 733 & 89 & 178 & & & & \\
\hline Paragominas & 18,9 & 48,0 & 76 & 195 & 729 & & & & \\
\hline
\end{tabular}

As fontes e as doses de nutrientes utilizadas no experimento foram: $\mathrm{N}-150$ $\mathrm{mg} \mathrm{kg}^{-1}$ de solo - uréia; $\mathrm{P}_{2} \mathrm{O}_{5}-200 \mathrm{mg} \mathrm{kg}^{-}$ ${ }^{1}$ de solo - fosfato de sódio; $\mathrm{K}_{2} \mathrm{O}-100 \mathrm{mg}$ $\mathrm{kg}^{-1}$ - sulfato de potássio; $\mathrm{Ca}-200 \mathrm{mg} \mathrm{kg}$ 1 - cloreto de cálcio; $\mathrm{Mg}-50 \mathrm{mg} \mathrm{kg}{ }^{-1}$ Cloreto de magnésio; $\mathrm{S}-40 \mathrm{mg} \mathrm{kg}{ }^{-1}-$ Sulfato de sódio; B - 1,0 mg kg-1 - ácido bórico; $\mathrm{Zn} \mathrm{-} \mathrm{5,0} \mathrm{mg} \mathrm{kg}^{-1}$ - Sulfato de zinco; $\mathrm{Cu}-1,0 \mathrm{mg} \mathrm{kg}^{-1}$ - Sulfato de cobre; $\mathrm{Si}-200 \mathrm{mg} \mathrm{kg}^{-1}$ - Silicato de cálcio e Ni $2 \mathrm{mg} \mathrm{kg}^{-1}$ - cloreto de níquel.

Avaliou-se no trabalho as variáveis: altura da planta de arroz e o número de perfilhos em três períodos distintos (vegetativo, florescimento e enchimento de grãos).

Os dados obtidos foram submetidos ao teste de normalidade e homocedasticidade, em seguida, os resultados foram analisados pelo teste Dunnett $(\mathrm{p}<0,05)$, utilizando o software estatístico Assistat.

Para a variável altura da planta, houve diferença significativa nas três leituras nos tratamentos omissão de fósforo e controle em relação ao completo, no solo proveniente de LATOSSOLO AMARELO de textura média (Capitão Poço).

O tratamento omissão de fósforo obteve valor inferior ao tratamento controle. Enquanto, no solo proveniente do município de Paragominas, o LATOSSOLO AMARELO de textura argilosa, o tratamento omissão de $\mathrm{K}$ diferiu significativamente do tratamento completo nas três leituras.

Além disso, por meio da comparação das médias entre as leituras, verificou-se 
diferença significativa nos tratamentos, em que os maiores resultados são apresentados na terceira leitura (enchimento de grãos), nos solos advindos dos dois locais.
$\mathrm{Na}$ terceira leitura não foi observado diferença significativa entre os solos avaliados, com exceção do tratamento omissão de Ni (Tabela 2).

Tabela 2. Altura da planta de arroz em três períodos de avaliação, 1LET (vegetativo), 2 LET (florescimento) e 3 LET (enchimento de grãos) nos solos coletados dos municípios de Paragominas (Latossolo Amarelo de textura argilosa) e Capitão Poço (Latossolo Amarelo de textura média), Estado do Pará.

\begin{tabular}{|c|c|c|c|c|c|c|}
\hline \multirow[t]{2}{*}{ TRAT } & \multicolumn{3}{|c|}{ Paragominas } & \multicolumn{3}{|c|}{ Capitão Poço } \\
\hline & 1LET & 2LET & 3LET & 1LET & 2LET & 3LET \\
\hline $\mathrm{T} 1$ & $77,33 \mathrm{Bb}$ & $105,00 \mathrm{Aa}$ & $111,67 \mathrm{Aa}$ & $90,67 \mathrm{Ac}$ & $103,33 \mathrm{Ab}$ & $114,67 \mathrm{Aa}$ \\
\hline $\mathrm{T} 2$ & n.s. $81,33 \mathrm{Ac}$ & n.s. $100,67 \mathrm{Ab}$ & n.s. $114,67 \mathrm{Aa}$ & ${ }^{*} 78,00 \mathrm{Ab}$ & n.s. $105,67 \mathrm{Aa}$ & n.s. $107,33 \mathrm{Aa}$ \\
\hline $\mathrm{T} 3$ & n.s. $77,67 \mathrm{Bc}$ & n.s. $97,67 \mathrm{Ab}$ & n.s. $109,33 \mathrm{Aa}$ & n.s. $86,67 \mathrm{Ac}$ & n.s. $97,67 \mathrm{Ab}$ & n.s. $115,67 \mathrm{Aa}$ \\
\hline $\mathrm{T} 4$ & * $41,67 \mathrm{Bc}$ & $* 60,33 \mathrm{Bb}$ & *83,00Ba & ${ }^{*} 78,00 \mathrm{Ac}$ & n.s. $103,67 \mathrm{Ab}$ & n.s. $120,67 \mathrm{Aa}$ \\
\hline T5 & n.s. $78,67 \mathrm{Ac}$ & n.s. $95,00 \mathrm{Ab}$ & n.s. $114,00 \mathrm{Aa}$ & ${ }^{*} 79,67 \mathrm{Ab}$ & ${ }^{*} 90,67 \mathrm{Aa}$ & * $92,00 \mathrm{Ba}$ \\
\hline T6 & n.s. $79,67 \mathrm{Ab}$ & n.s. $106,00 \mathrm{Aa}$ & n.s. $107,00 \mathrm{Ba}$ & n.s. $84,67 \mathrm{Ac}$ & n.s. $103,33 \mathrm{Ab}$ & n.s. $114,67 \mathrm{Aa}$ \\
\hline $\mathrm{T} 7$ & n.s. $82,33 \mathrm{Ab}$ & n.s. $102,00 \mathrm{Aa}$ & n.s. $110,67 \mathrm{Ba}$ & n.s. $86,33 \mathrm{Ac}$ & n.s. $106,33 \mathrm{Ab}$ & n.s. $121,33 \mathrm{Aa}$ \\
\hline $\mathrm{T} 8$ & n.s. $79,00 \mathrm{Ac}$ & n.s. $102,67 \mathrm{Ab}$ & n.s. $113,67 \mathrm{Aa}$ & n.s. $80,67 \mathrm{Ac}$ & n.s. $103,00 \mathrm{Ab}$ & n.s. $116,00 \mathrm{Aa}$ \\
\hline T9 & n.s. $81,33 \mathrm{Ac}$ & n.s. $103,00 \mathrm{Ab}$ & n.s. $112,33 \mathrm{Aa}$ & n.s. $85,33 \mathrm{Ac}$ & n.s. $100,33 \mathrm{Ab}$ & n.s. $109,33 \mathrm{Aa}$ \\
\hline $\mathrm{T} 10$ & n.s. $79,67 \mathrm{Ac}$ & n.s. $97,33 \mathrm{Bb}$ & n.s. $108,67 \mathrm{Ba}$ & n.s. $85,00 \mathrm{Ac}$ & n.s. $106,67 \mathrm{Ab}$ & n.s. $119,00 \mathrm{Aa}$ \\
\hline $\mathrm{T} 11$ & ${ }^{*} 50,67 \mathrm{Ac}$ & ${ }^{*} 76,67 \mathrm{Ab}$ & *86,33Aa & ${ }^{*} 38,67 \mathrm{Bc}$ & * $57,67 \mathrm{Bb}$ & *80,67Aa \\
\hline C.V. $(\%)$ & 3,67 & 5,03 & 5,13 & 5,28 & 5,11 & 4,25 \\
\hline D.M.S. & 6,53 & 11,56 & 13,20 & 10,13 & 12,11 & 11,29 \\
\hline
\end{tabular}

" Médias seguidas por um asterisco na mesma coluna diferem significativamente do completo, ao nível de 5\% de probabilidade pelo teste de Dunnett.

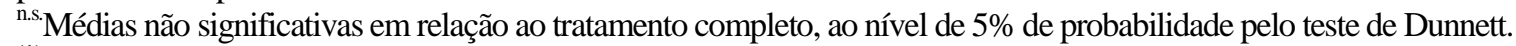

${ }^{(1)}$ Médias seguidas de mesma letra maiúscula (locais) e minúscula (período de avaliação) na linha, não diferem entre si a 5\% de probabilidade pelo teste F e de Tukey, respectivamente.

A deficiência de $\mathrm{P}$ proporcionou menor crescimento da planta, no solo proveniente de LATOSSOLO AMARELO de textura média (Capitão Poço), possivelmente, por ser o nutriente responsável pelo desenvolvimento radicular, principalmente, nas fases iniciais de crescimento (SCIVITTARO et al., 2006).

O Latossolo originado da cidade de Paragominas apresentou uma deficiência expressiva de $\mathrm{K}$, isto acarreta em baixo crescimento na cultura. Os sintomas iniciais dessa deficiência é a clorose branca nas pontas das folhas mais velhas e, à medida que a deficiência se intensifica, o tecido da folha torna-se marrom e necrótico, progredindo da ponta da folha para sua margem, desenvolvendo-se um amarelecimento no formato de "V" invertido (VELOSO et al., 2009).

Para a variável número de perfilhos por planta, nas duas últimas leituras houve diferença significativa apenas nos tratamentos omissão de fósforo e controle 
em relação ao completo, nos solos provenientes de LATOSSOLO AMARELO de textura média (Capitão Poço) e LATOSSOLO AMARELO de textura argilosa (Paragominas). Com relação às médias obtidas nas leituras, não se observou diferença significativa nas duas últimas leituras em todos os tratamentos, no solo proveniente de LATOSSOLO AMARELO de textura argilosa (Paragominas) (Tabela $3)$.

Tabela 3. Número de perfilhos por planta de arroz em três períodos de avaliação, 1LET (vegetativo), 2 LET (florescimento) e 3 LET (enchimento de grãos) nos solos coletados dos municípios de Paragominas (Latossolo Amarelo de textura argilosa) e Capitão Poço (Latossolo Amarelo de textura média), Estado do Pará.

\begin{tabular}{|c|c|c|c|c|c|c|}
\hline \multirow[t]{2}{*}{ TRAT } & \multicolumn{3}{|c|}{ Paragominas } & \multicolumn{3}{|c|}{ Capitão Poço } \\
\hline & 1LET & 2LET & 3LET & 1LET & 2LET & 3LET \\
\hline $\mathrm{T} 1$ & $0,40 \mathrm{Ab}$ & $2,20 \mathrm{Aa}$ & $2,07 \mathrm{Aa}$ & $0,20 \mathrm{Ab}$ & $2,27 \mathrm{Aa}$ & $1,80 \mathrm{Aa}$ \\
\hline $\mathrm{T} 2$ & n.s. $0,93 \mathrm{Ab}$ & n.s. $2,60 \mathrm{Aa}$ & n.s. $2,07 \mathrm{Aa}$ & n.s. $0,27 \mathrm{Ab}$ & n.s. $2,20 \mathrm{Aa}$ & n.s. $2,00 \mathrm{Aa}$ \\
\hline T3 & n.s. $0,07 \mathrm{Ab}$ & n.s. $2,27 \mathrm{Aa}$ & n.s. $1,60 \mathrm{Aa}$ & n.s. $0,00 \mathrm{Aa}$ & ${ }^{*} 0,00 \mathrm{Ba}$ & ${ }^{*} 0,00 \mathrm{Ba}$ \\
\hline $\mathrm{T} 4$ & n.s. $0,00 \mathrm{Aa}$ & *0,00Ba & ${ }^{*} 0,00 \mathrm{Ba}$ & n.s. $0,00 \mathrm{Ab}$ & *1,40Aa & n.s. $1,27 \mathrm{Aa}$ \\
\hline $\mathrm{T} 5$ & n.s. $0,13 \mathrm{Ab}$ & n.s. $2,60 \mathrm{Aa}$ & n.s. $2,13 \mathrm{Aa}$ & n.s. $0,00 \mathrm{Ab}$ & n.s. $1,87 \mathrm{Ba}$ & n.s. $1,13 \mathrm{Ba}$ \\
\hline T6 & n.s. $0,53 \mathrm{Ab}$ & n.s. $2,40 \mathrm{Aa}$ & n.s. $2,40 \mathrm{Aa}$ & n.s. $0,47 \mathrm{Ab}$ & n.s. $1,93 \mathrm{Aa}$ & n.s. $1,13 \mathrm{Bab}$ \\
\hline $\mathrm{T} 7$ & n.s. $0,60 \mathrm{Ab}$ & n.s. $2,47 \mathrm{Aa}$ & n.s. $2,33 \mathrm{Aa}$ & n.s. $0,33 \mathrm{Ab}$ & n.s. $2,00 \mathrm{Aa}$ & n.s. $1,13 \mathrm{Bb}$ \\
\hline $\mathrm{T} 8$ & n.s. $0,13 \mathrm{Ab}$ & n.s. $2,33 \mathrm{Aa}$ & n.s. $1,87 \mathrm{Aa}$ & n.s. $0,07 \mathrm{Ab}$ & n.s. $2,07 \mathrm{Aa}$ & n.s. $1,40 \mathrm{Aa}$ \\
\hline T9 & n.s. $0,13 \mathrm{Ab}$ & n.s. $3,00 \mathrm{Aa}$ & n.s. $2,27 \mathrm{Aa}$ & n.s. $0,07 \mathrm{Ac}$ & n.s. $2,73 \mathrm{Aa}$ & n.s. $1,87 \mathrm{Ab}$ \\
\hline $\mathrm{T} 10$ & n.s. $0,27 \mathrm{Ab}$ & n.s. $2,80 \mathrm{Aa}$ & n.s. $2,40 \mathrm{Aa}$ & n.s. $0,40 \mathrm{Ab}$ & n.s. $2,00 \mathrm{Ba}$ & n.s. $1,80 \mathrm{Aa}$ \\
\hline $\mathrm{T} 11$ & n.s. $0,00 \mathrm{Aa}$ & *0,07Aa & $0,07 \mathrm{Aa}$ & n.s. $0,00 \mathrm{Aa}$ & * $0,00 \mathrm{Aa}$ & *0,00Aa \\
\hline C.V. (\%) & 14,07 & 16,62 & 12,73 & 9,50 & 7,62 & 9,74 \\
\hline D.M.S. & 0,29 & 0,62 & 0,45 & 0,18 & 0,26 & 0,30 \\
\hline
\end{tabular}

* Médias seguidas por um asterisco na mesma coluna diferem significativamente do completo, ao nível de 5\% de probabilidade pelo teste de Dunnett.

${ }_{\text {n.s. }}$ Médias não significativas em relação ao tratamento completo, ao nível de $5 \%$ de probabilidade pelo teste de Dunnett.

${ }^{(1)}$ Médias seguidas de mesma letra maiúscula (locais) e minúscula (período de avaliação) na linha, não diferem entre si a 5\% de probabilidade pelo teste F e de Tukey, respectivamente.

Em relação ao advindo de LATOSSOLO AMARELO de textura média (Capitão Poço) houve diferença significativa entre as duas últimas leituras nos tratamentos omissão de $\mathrm{Ca}$, de $\mathrm{Mg}$ e de B, $\mathrm{Zn}, \mathrm{Cu}, \mathrm{Si}$ e Ni. Entre os solos, na última leitura ocorreu resultados diferentes nos tratamentos omissão de $\mathrm{N}$, de $\mathrm{P}$, de $\mathrm{K}$, de $\mathrm{Ca}$ e de Mg. No Latossolo de Paragominas, a menor resposta, na terceira avaliação foi no tratamento omissão de $\mathrm{P}$ e no de Capitão Poço foi na omissão de N.

A deficiência do fósforo ocasionou menor crescimento da planta no LATOSSOLO AMARELO de textura média, enquanto em LATOSSOLO AMARELO de textura argilosa a omissão de potássio proporciona o menor crescimento do arroz. 


\section{REFERÊNCIAS}

FAGERIA, N. K.; SLATON, N. A.;

BALIGAR, V. C. Nutrient management for improving lowland rice productivity and sustainability. Advances in Agronomy, v. 80, p. 63-152, 2003.

GONDIM A. R. O.; PRADO, R. M.; ALVES, A. U.; FONSECA, I. M. Eficiência nutricional do milho cv. BRS 1030 submetido à omissão de macronutrientes em solução nutritiva. Revista Ceres, v. 57, n. 4, p. 539-544, 2010.

HERNANDES, A.; BUZETTI, S.; ANDREOTTI, M.; ARF, O.; SÁ, M. E. Doses, fontes e épocas de aplicação de nitrogênio em cultivares de arroz. Ciência e Agrotecnologia, v. 34, n. 2, p. 307-312, 2010.

MAUAD, M.; GRASSI FILHO, H.; CRUSCIOL, C. A. C.; CORRÊA, J. C. Teores de silício no solo e na planta de arrozde terras altas com diferentes doses deadubação silicatada e nitrogenada. Revista Brasileira de Ciência do Solo, v. 27, p. 867-873, 2003.

SCIVITTARO, W. B. Otimização do uso de fertilizantes nas lavouras de arroz do RS. In: GOMES, A. S.; PETRINI, J. A.; FAGUNDES, P. R. R. Manejo racional da cultura do arroz irrigado "Projeto
Marca". Pelotas, Embrapa Clima Temperado, 2006. p.77-90.

VELOSO, C. A. C.; BOTELHO, S. M. B.; LOPES, A. M.; CARVALHO, E. J. M. Nutrição mineral e adubação da cultura do arroz de sequeiro. Belém, PA: Embrapa Amazônia Oriental, 2009. 29 p. (Embrapa Amazônia

Oriental.

Documentos, 360).

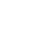

